\title{
Sex-related differences in outcome after endovascular revascularization for lower extremity artery disease
}

\section{A single-centre analysis of a specialized vascular unit}

\author{
Henrike Barenbrock ${ }^{1}$, Jannik Feld ${ }^{2}$, Antonia Lakomek ${ }^{1}$, Kristina Volkery ${ }^{1}$, \\ Jeanette Köppe ${ }^{2}$, Lena Makowski ${ }^{1}$, Christiane M. Engelbertz ${ }^{1}$, Holger Reinecke ${ }^{1}$, \\ Nasser Malyar ${ }^{1}$, and Eva Freisinger ${ }^{1}$ \\ Division of Vascular Medicine, Department of Cardiology I, Coronary and Peripheral Vascular Disease, Heart Failure, \\ University Hospital Muenster, Germany \\ ${ }^{2}$ Institute of Biostatistics and Clinical Research, University of Muenster, Germany
}

\begin{abstract}
Summary: Background: Sex-related differences may influence the outcome of endovascular revascularization (EVR) in patients with lower extremity arterial disease (LEAD) even under optimized healthcare supply. Patients and methods: LEAD patients who underwent EVR at the Department of Cardiology I - Coronary and Peripheral Vascular Disease, Heart Failure, University Hospital Muenster, Germany between 2014 and 2016 were included into the retrospective study. Detailed information on risk factors and co-morbidities, medication, LEAD related measures, and interventional parameters were assessed. Outcome defined as technical success rate, complications, and mortality was analyzed up to 12 months follow-up. Results: In total, 165 female and 437 male LEAD patients were included. Women and men presented with comparable severity of LEAD in terms of critical limb threatening ischemia (46.2\%), wound status (34.9\%), and amputation rate (9.6\%, all n.s.) at index. Intake of platelet inhibitors (65.8\% female vs. $70.0 \%$ male), oral anticoagulants (21.3\% vs. $25.4 \%$ ), and statins $(65.6 \%$ vs. $76.0 \%)$ was observed less frequently in female patients. Against the background of high technical success (85\%), inhospital death (0.8\%), severe adverse cardiac (MCE; 1.7\%), and limb events (MALE; $6.1 \%$ ) occurred at low rates in either sex. Adjusted long-term mortality was not affected by patients' sex (female HR 0.755; $p=0.312$ ). Conclusions: Despite critical LEAD stages in every second patient, EVR was performed safe with high technical success rates in female and male patients. Longterm outcomes were observed at comparatively low rates in both sexes at the specialized vascular center. During aftercare, supply with statin therapy turned out improvable particularly in female LEAD patients.
\end{abstract}

Keywords: lower extremity artery disease, sex differences, cardiovascular risk factors, clinical outcome, endovascular revascularization

\section{Introduction}

Lower extremity artery disease (LEAD) can mostly be attributed to atherosclerosis leading to peripheral artery obstruction with reduced blood flow and limb ischemia. Atherosclerosis is a systemic disease which often leads to simultaneous lesions in other vascular beds $[1,2]$. The risk factors that are associated with the development of LEAD are principally similar to those that favour the development of chronic coronary syndrome (CCD) [1]. Age, obesity, smoking, diabetes, arterial hypertension, dyslipidemia, and chronical kidney disease (CKD) are common risk factors and co-morbidities that promote atherosclerosis and LEAD [1, 3, 4]. However, studies could identify deviating impact of risk factors on LEAD compared to atherosclerotic manifestations in other vascular beds [3, 4]. Amongst others, there is evidence for sex-related differences in risk constellation and progression of LEAD [5]. Women are reported to present less frequently with traditional cardiovascular risk factors $[5,6,7]$, but are typically at older ages at the time of diagnosis compared to male LEAD patients $[5,6,7,8,9]$. Women are reported to have less classical symptoms [5] which may contribute to a higher prevalence of critical limb threatening ischemia (CLTI) at the time of treatment initiation [6]. In cardiovascular disease, female patients have been indicated an underuse of pharmacological [10] and interventional therapy [11]. However, in the context of LEAD the impact of patients' sex on treatment 
supply is not well studied. Moreover, there is contradictory data on the impact of patients' sex on short- and long-term risk of amputations and mortality after endovascular revascularization (EVR) for LEAD [7, 8, 9, 11, 12, 13, 14]. Given the worldwide rising prevalence of LEAD in both sexes, evaluating the reality of treatment supply and its impact on clinical outcome in unselected patient cohorts focusing sex-related differences is of increasing importance. However, randomized clinical trials (RCTs) do not represent daily clinical practice due to highly preselected patients leading to embellished clinical outcome [15]. On the other hand, nationwide data evaluating the reality of treatment supply and outcome may underestimate the opportunities that lie within optimal guideline-conform patient care [16].

Therefore, the aim of our study was to investigate sexrelated treatment and outcome predictors after EVR in a specialized university vascular unit representing high standard health care.

\section{Patients and methods}

The retrospective study cohort comprised all LEAD patients of the Department of Cardiology I - Coronary and Peripheral Vascular Disease, Heart Failure, University Hospital Muenster, Germany, who received EVR between 1st of January 2014 and 31st of December 2016. In total, 602 LEAD patients with index EVR were enrolled, thereof $\mathrm{n}=437(72.6 \%)$ male and $\mathrm{n}=167(27.4 \%)$ female patients. Comprehensive clinical data were obtained at index hospitalization, and at ambulatory follow-up visits at 6 months, and at 12 months after index EVR. These comprised detailed information on LEAD status (e.g. Fontaine stage, ankle-brachial-index (ABI), walking distance, wound status, amputation status), the presence of co-morbidities, cardiovascular risk factors and atherosclerotic manifestation, drug therapy, and laboratory parameters as well as detailed information on EVR index procedure. Survival was followed at the latest until 1st of July 2019.

\section{Diagnostic and procedural parameters}

LEAD was defined by ABI and clinical parameters and was staged according to Fontaine classification. Critical limb threatening ischemia (CLTI) was defined as Fontaine stages III (rest pain) and IV (ulcer/ gangrene), non-CLTI was defined as Fontaine stages I (symptom free), IIa (intermittent claudication, IC $>200 \mathrm{~m}$ ), and IIb (IC<200 m). To assess ABI, systolic blood pressures in brachial, posterior, and anterior tibial arteries on both sides were obtained and ABI-values for each leg were calculated conform with current guidelines [17].

Cardiovascular risk factors and co-morbidities were assessed based on information of standardized patient files and findings reports using the hospital information system. These diagnostic information comprised in detail: chronic coronary syndrome (CCS; including previous myocardial infarction), cerebrovascular disease (CVD; including previous stroke), visceral and renal artery disease (VAD), chronic kidney disease (CKD), chronic heart failure (CHF), atrial fibrillation/ flutter, diabetes, arterial hypertension, dyslipidemia, body mass index (BMI), age, smoking (active and previous), and malignant diseases. Technical details of EVR including contrast volume, duration of the procedure, target vessel and side, puncture site, sheath size, and catheters applied were obtained from the catheter report. Further, aside from primary technical success, any conspicuities and complications during and after EVR (e.g. (micro-)embolism, prolonged or excessive bleeding or the need of blood transfusion, complications at puncture site, bailout surgery) were recorded. All-cause mortality, major adverse limb (MALE; re-EVR, bypass, or amputation of the lower limb [LL] treated at index), and cardiac events (MCE; acute coronary syndrome, percutaneous coronary intervention, coronary artery bypass graft, stroke) were defined as primary outcome parameters. To assess patients' vital status at 6 and 12 months FU, for patients without any further contact with our vascular division (follow-up visit, later intervention, hospital stay, or any other documented ambulatory or in-patient contact), the electronic patient file was checked for cross-departmental hospital contact, or if the patient was put "deceased" on the electronic patient file. All patients with still unclear vital status were then contacted by phone, or - if not available their general practitioner was contacted. For deceased patients, the date of death was obtained in order to perform Kaplan Meier analysis for accurate survival curves.

\section{Statistics}

Two-sided Chi-Quadrat-test for categorical variables and two-sided Fischer's exact test were used to test differences between male and female sex. Continuous variables were evaluated with two-sided Mann-Whitney-U-test. To compare differences on overall survival between both sexes for non-CLTI and CLTI patients separately, Kaplan-Meier estimators were calculated in both subgroups and for both sexes, and tested via two-sided log-rank test. To determine the effect of sex on overall survival, a multivariable Cox-regression analysis was performed adjusting for age, polyvascular disease, and CLTI as the main influencing variables. All analyses were fully explorative without adjustment for multiple test problem (hypothesis generating). Accordingly, $\mathrm{p}$-values $\mathrm{p}<0.05$ were interpreted as statistically noticeable. Data were collected by using hospital information system ORBIS $^{\circledR}$ Agfa Healthcare, edited with Microsoft Excel ${ }^{\circledR}$ (Microsoft Office Professional Plus), and were analyzed via IBM SPSS ${ }^{\circledR}$ Statistics Version 25 and R version 3.6.0 (2019-04-26), R foundation, Vienna, Austria.

\section{Results}

In total, $\mathrm{n}=602 \mathrm{LEAD}$ patients with index EVR between 01.01.2014 and 31.12.2016 were identified for further analysis, thereof $n=165 \quad(27.4 \%)$ female and $n=437$ 
Table I. Baseline characteristics at index EVR

\begin{tabular}{|c|c|c|c|c|}
\hline & Female & Male & All & P-value \\
\hline Patient number - n (\%) & $165(27.4)$ & $437(72.6)$ & $602(100)$ & $<0.001$ \\
\hline Age - years $\pm S D$ & $73.1 \pm 11.9$ & $68.9 \pm 10.1$ & $70.1 \pm 10.8$ & $<0.001$ \\
\hline $\mathrm{BMl}-\mathrm{kg} / \mathrm{m}^{2} \pm \mathrm{SD}$ & $25.07 \pm 5.27$ & $27.54 \pm 4.56$ & $26.87 \pm 4.89$ & $<0.001$ \\
\hline \multicolumn{5}{|l|}{ LEAD stages } \\
\hline LEAD Fon I - n (\%) & $2(1.3)$ & $1(0.2)$ & $3(0.5)$ & \multirow[t]{5}{*}{0.102} \\
\hline LEAD Fon Ila $-\mathrm{n}(\%)$ & $7(4.3)$ & $30(7.0)$ & $37(6.3)$ & \\
\hline LEAD Fon IIb - n (\%) & $65(40.6)$ & $208(48.7)$ & $273(46.5)$ & \\
\hline LEAD Fon III - n (\%) & $26(16.3)$ & $46(10.8)$ & $77(12.3)$ & \\
\hline LEAD Fon IV - n (\%) & $60(36.4)$ & $141(32.3)$ & $201(33.4)$ & \\
\hline CLTI - n (\%) & $86(52.1)$ & $187(42.8)$ & $278(46.2)$ & 0.050 \\
\hline Ipsilateral ABI in IC* - mean $\pm S D$ & $0.487( \pm 0.181)$ & $0.434( \pm 0.211)$ & $0.445( \pm 0.206)$ & 0.147 \\
\hline Ipsilateral $A B \mid$ in $\left.C L T\right|^{*}-$ mean $\pm S D$ & $0.382( \pm 0.230)$ & $0.379( \pm 0.298)$ & $0.380( \pm 0.279)$ & 0.734 \\
\hline Previous amputation** $-\mathrm{n}(\%)$ & $14(8.6)$ & $43(10.0)$ & $57(9.6)$ & 0.716 \\
\hline Wound status at index** $-n(\%)$ & $61(37.4)$ & $147(33.9)$ & $208(34.9)$ & 0.486 \\
\hline \multicolumn{5}{|l|}{ Arteriosclerosis manifestation } \\
\hline Chronic coronary syndrome - n (\%) & $62(38.3)$ & $264(61.1)$ & $326(54.9)$ & $<0.001$ \\
\hline Cerebrovascular disease $-\mathrm{n}(\%)$ & $34(21.0)$ & $147(34.3)$ & $181(30.6)$ & 0.002 \\
\hline Previous stroke - n (\%) & $14(8.6)$ & $63(14.7)$ & $77(13.0)$ & 0.071 \\
\hline Visceral artery disease $-\mathrm{n}(\%)$ & $11(6.8)$ & $24(5.6)$ & $35(5.9)$ & 0.719 \\
\hline Polyvascular disease $-n(\%)^{\star \star \star}$ & $81(50.0)$ & $307(71.2)$ & $388(65.4)$ & $<0.001$ \\
\hline \multicolumn{5}{|l|}{ Cardiovascular comorbidity } \\
\hline Atrial fibrillation/flutter - n (\%) & $37(22.8)$ & $118(27.3)$ & $155(26.1)$ & 0.317 \\
\hline Chronic Heart Failure - n (\%) & $32(19.9)$ & $87(20.2)$ & $119(20.1)$ & 1.000 \\
\hline Chronic Kidney Disease - n (\%) & $45(27.8)$ & $123(28.5)$ & $168(28.3)$ & 0.948 \\
\hline Diabetes, type 1 and $2-n(\%)$ & $50(30.7)$ & $176(40.7)$ & $226(38.0)$ & 0.031 \\
\hline Dyslipidemia - n (\%) & $81(50.0)$ & $256(59.7)$ & $337(57.0)$ & 0.043 \\
\hline Hypertension - n (\%) & $128(79.0)$ & $378(87.9)$ & $506(85.4)$ & 0.009 \\
\hline Smoking, active or previous - $\mathrm{n}(\%)$ & $83(51.2)$ & $292(67.4)$ & $375(63.0)$ & $<0.001$ \\
\hline Cancer - n (\%) & $25(15.4)$ & $68(15.8)$ & $93(15.7)$ & 1.000 \\
\hline
\end{tabular}

Notes. EVR: endovascular revascularization; LEAD: lower extremity artery disease; FON: Fontaine stage; IC: intermittent claudication; CLTI: chronic limb threatening ischemia; ABI: ankle brachial index. *Lower ABI (ATA; ATP) of the treated side at index; without amputees; value for mediasclerosis was set zero. ** Previous amputation and wound status index - ipsi- and/or contralateral. *** Polyvascular disease - besides LEAD at least one further of the following: chronic coronary syndrome, cerebrovascular disease, visceral artery disease.

(72.6\%) male patients. Female patients were on average 4 years older compared to males. At the time of index EVR, female and male LEAD presented with comparable severity as indicated by LEAD stages and CLTI rate (52\% vs. $43 \%, \mathrm{p}=0.050$ ). One-third of patients presented with a chronic wound at index, and one in ten patients had previous amputation of the lower limbs, however with no difference between both sexes.

In terms of risk profiles, female patients were observed less often to present with arteriosclerotic manifestations other than LEAD (polyvascular disease; 50\% vs. $71 \%$ ) as they had lower rate of chronic coronary syndrome $(38 \%$ vs. $61 \%)$ and cerebrovascular disease (21\% vs. $34 \%$, all $\mathrm{p}<0.01$ ) than male LEAD patients (Table I). Most common cardiovascular risk factors were arterial hypertension (85\%), smoking (63\%), and dyslipidemia (57\%). Particularly, diabetes ( $31 \%$ vs. $41 \%, \mathrm{p}=0.031)$, arterial hypertension (79\% vs. $88 \%, \mathrm{p}=0.009)$, dyslipidemia (50\% vs. $60 \%, \mathrm{p}=0.043)$, and smoking ( $51 \%$ vs. $67 \%, \mathrm{p}<0.001)$ were more frequent in male patients whereas atrial fibrillation, chronic heart failure, or chronic kidney disease did not differ between the sexes.

\section{EVR and primary Outcome}

Index EVR did not differ in terms of procedural details: Irrespective of patients' sex, treated vascular segments were most commonly femoro-popliteal (76\%), followed by infrapopliteal (39\%), iliac (27\%), and bypass interventions (4\%, Table II). Almost all patients underwent plain old balloon angioplasty (POBA $96 \%$ of patients). Stent EVR (BMS; $57 \%$ vs. 65\%), as well as the usage of drugcoated devices (DES; 9\% vs. 7\%; DCB 38\% vs. 37\%; all n.s.) applied with equal frequency in female and male patients. Overall, primary technical success of EVR was high and did not differ between both sexes (87\% vs. 84\%, n.s.; Table II). Peri-procedural complications including minor complications (e.g. minor bleeding at puncture site, adaptation of vascular access) occurred similarly frequent in female and male patients ( $20 \%$ vs. $19 \%$, n.s.). Also, the rate of post-interventional complications at puncture site (e.g. local bleeding, arteriovenous fistula, aneurysm, surgery) did not differ notably (21\% vs. $15 \%$, n.s.). Severe complications occurred in a single-digit percentage area in both sexes: There was no difference in blood transfusion rate 
Table II. EVR parameters and observed outcome

\begin{tabular}{|c|c|c|c|c|}
\hline & Female & Male & All & P-value \\
\hline \multicolumn{5}{|l|}{ Intervention } \\
\hline EVR duration - minutes $( \pm S D)$ & $92.1 \pm 44.0$ & $98.4 \pm 46.0$ & $96.6 \pm 45.5$ & 0.127 \\
\hline EVR contrast medium - volume $( \pm S D)$ & $121.4 \pm 53.2$ & $125.2 \pm 48.9$ & $124.2 \pm 50.1$ & 0.481 \\
\hline \multicolumn{5}{|l|}{ EVR treated arterial segment* } \\
\hline Bypass segment - n (\%) & $5(3.0)$ & $19(4.4)$ & $24(4.0)$ & 0.611 \\
\hline Iliac segment - n (\%) & $47(28.7)$ & $112(25.7)$ & $159(26.5)$ & 0.528 \\
\hline Femoro-popliteal segment - n (\%) & $123(75.0)$ & $333(76.4)$ & $456(76.0)$ & 0.807 \\
\hline Infrapopliteal segment - n (\%) & $60(36.7)$ & $172(39.4)$ & $232(38.7)$ & 0.584 \\
\hline \multicolumn{5}{|l|}{ EVR devices* } \\
\hline DES - n (\%) & $15(9.1)$ & $32(7.3)$ & $47(7.8)$ & 0.573 \\
\hline $\mathrm{DCB}-\mathrm{n}(\%)$ & $63(38.4)$ & $163(37.4)$ & $226(37.6)$ & 0.891 \\
\hline BMS - n (\%) & $93(56.7)$ & $284(65)$. & $377(62.8)$ & 0.070 \\
\hline POBA - n (\%) & $150(92.0)$ & $422(97.0)$ & $572(95.7)$ & 0.015 \\
\hline \multicolumn{5}{|l|}{ EVR outcome } \\
\hline EVR primary technical success - n (\%) & $144(87.3)$ & $368(84.4)$ & $512(85.2)$ & 0.773 \\
\hline Peri-procedural complication - n (\%)** & $32(19.6)$ & $81(18.6)$ & $113(18.9)$ & 0.870 \\
\hline 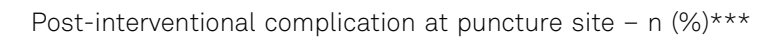 & $34(21.3)$ & $62(14.6)$ & $96(16.4)$ & 0.069 \\
\hline Blood transfusion - n (\%) & $5(3.2)$ & $8(1.9)$ & $13(2.2)$ & 0.554 \\
\hline MALE - n (\%) & $8(5.0)$ & $28(6.6)$ & $36(6.1)$ & 0.596 \\
\hline In-hospital amputation, ipsilateral - n (\%) & $3(1.9)$ & $15(3.5)$ & $18(3.1)$ & 0.441 \\
\hline MCE - n (\%) & $4(2.5)$ & $6(1.4)$ & $10(1.7)$ & 0.592 \\
\hline In-hospital mortality - n (\%) & $2(1.2)$ & $3(0.7)$ & $5(0.8)$ & 0.896 \\
\hline
\end{tabular}

Notes. BMS: Bare metal stent; DES: drug-eluting stent; POBA: plain old balloon angioplasty; DCB: drug-coated balloon; MCE: major adverse cardiac events; MALE: major adverse limb event. *Multiple mention possible. ${ }^{*}$ Peri-procedural complication - including (micro-)embolism, (minor and major) bleeding, necessity of another access path. ${ }^{* *}$ Post-interventional complication at puncture side - including aneurysm, arteriovenous fistula, local bleeding, necessity of surgery.

(3\% vs. $2 \%$, n.s.), major adverse limb events (MALE; recurrent EVR, bypass and / or limb amputation; $5 \%$ vs. $7 \%$, n.s.), and major adverse cardiac events (MCE; acute coronary syndrome, percutaneous coronary intervention, coronary artery bypass graft, stroke; $3 \%$ vs. $1 \%$, n.s.). There were only five cases of in-hospital death in the entire patient cohort $(0.8 \%)$.

\section{Long-term Outcome}

Up to 12 month, $6.2 \%$ of female and $5.3 \%$ of male LEAD patients have died (electronic supplementary material [ESM] 1). Survival curves of male and female LEAD patients depending on CLTI status show 1-year survival to be $98.6 \%$ in non-CLTI (Figure 1A) compared to $89.0 \%$ in CLTI (Figure 1B). However, after adjustment for age, the presence of polyvascular disease and CLTI, overall survival did not differ between the sexes (female sex HR 0.755; 95\%-CI: 0.437-1.303, n.s.; Figure 2). Of these factors, presence of CLTI was the strongest predictor of death in either sex (female HR 4.73; male HR 3.17; both $\mathrm{p}<0.05$ ). The frequency of severe adverse events was reported at low frequencies at 6 and 12 months follow-up visits (ESM 1). In the first half-year period from index, $16.1 \%$ of patients experienced MALE, thereof $11.1 \%$ re-intervention, $0.7 \%$ vascular surgery, and 2.1\% ipsilateral amputation. At 12 months FU, $15.1 \%$ of patients reported MALE, thereof 9.1\% re-intervention, $0.3 \%$ vascular surgery, and $0.8 \%$ ipsilateral amputation. MCE was reported in $2.8 \%$ of patients at 6 months and $3.1 \%$ at 12 months FU visits. Amongst those patients that survived one year from index EVR, a clinically relevant improvement of ABI could be shown in both sexes (ESM 2). Sub-analysis comparing LEAD patients' ABI values at index and 12 months FU ( $n=290$ patients, $28 \%$ female; ESM 2) showed sustained improvement of ABI category after EVR. At index, onequarter of patients had critical ABI values $<0.5$, one-third presented with $\mathrm{ABI} 0.5-<0.75$, and $\mathrm{ABI}$ values $>1.3$ corresponding to media sclerosis were determined in $7 \%$ of patients. After 12 months, the number of patients with highly critical perfusion decreased to $16 \%$ in female and $19 \%$ in male patients. The rate of patients with normal ABI values at 12 months follow-up visits increased in male but not female patients (male $18 \%$ at index vs. $24 \%$ at $12 \mathrm{M})$.

\section{Pharmacotherapy}

At index, $82 \%$ of female and $86 \%$ of male patients received at least one antithrombotic drug (either platelet aggregation inhibitor; PAI or oral anticoagulant; OAC; ESM 3). At discharge after EVR, its usage increased to $100 \%$ in both sexes. Among patients adherent to FU visits, antithrombotic therapy remained almost complete (99\% female, $98 \%$ male at $12 \mathrm{M})$. Considering OAC separately, $29 \%$ of female and $23 \%$ of male patients received OAC at 

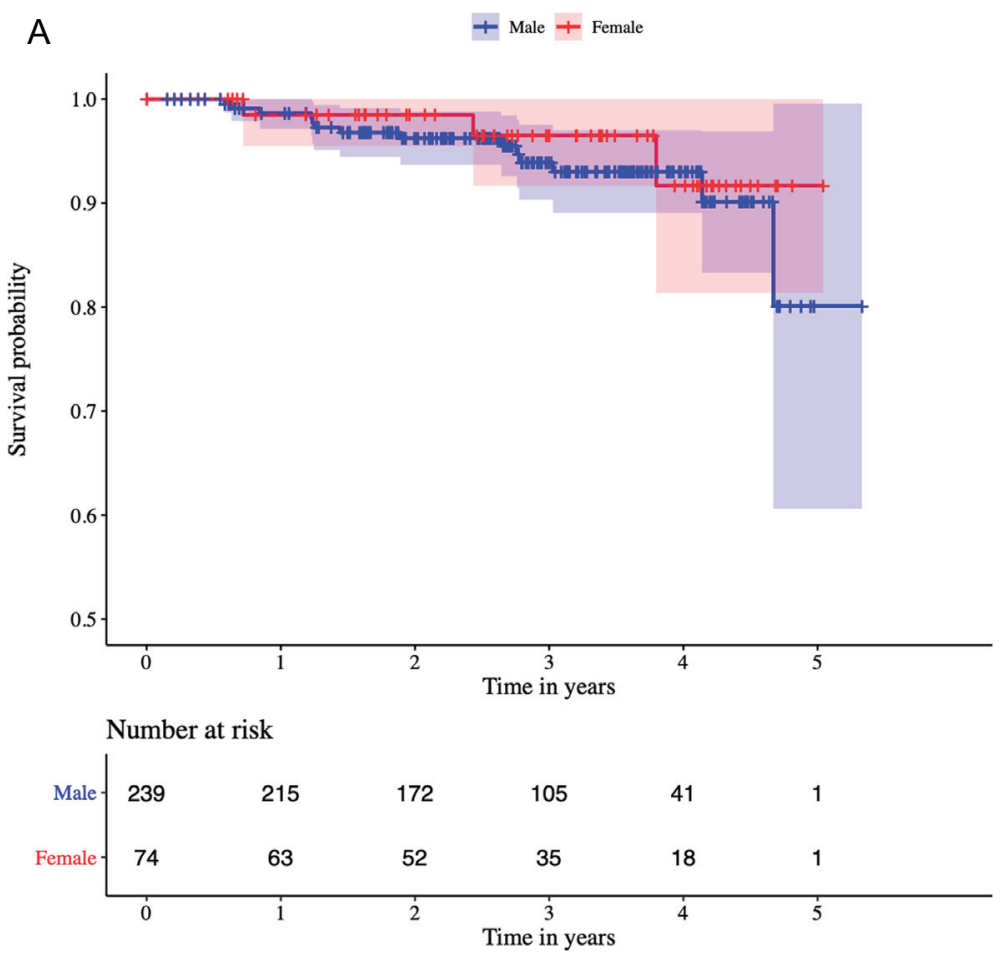

B

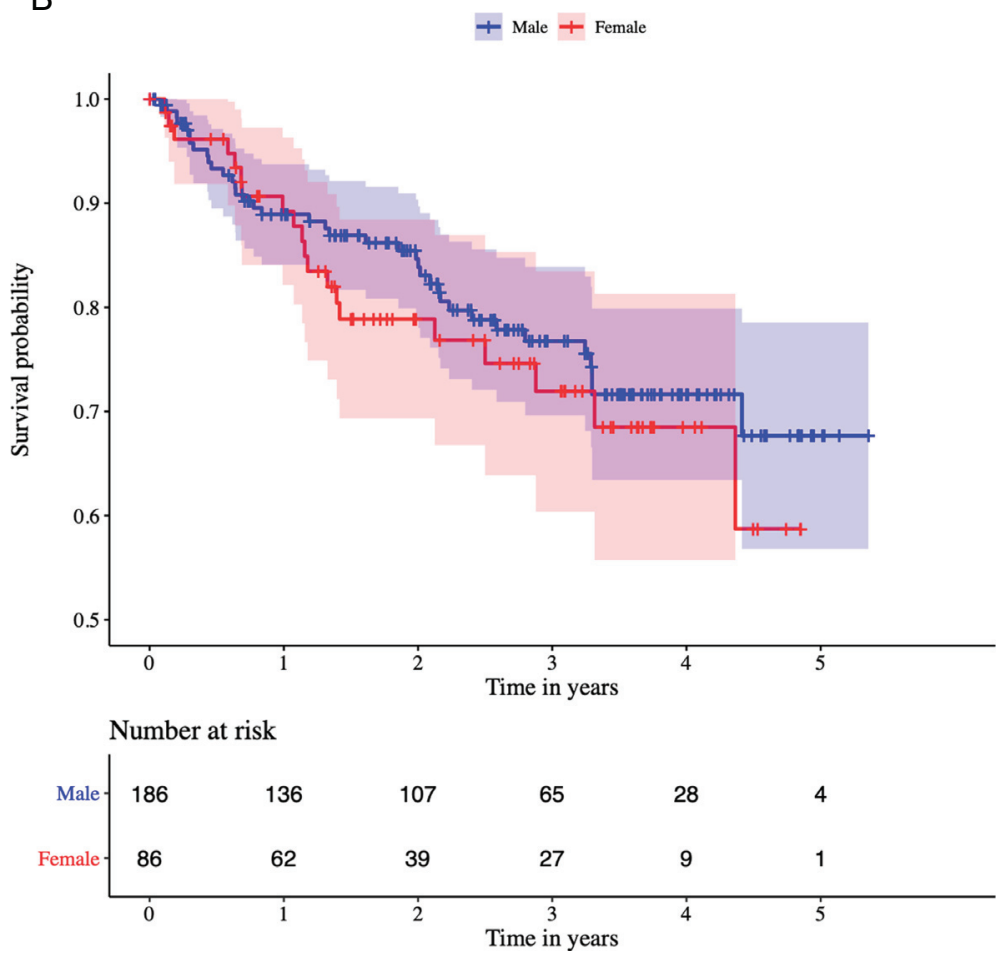

Figure 1. Survival curves of LEAD patients with and without CLTI separated by male and female sex. Panel A shows survival curves of female (red) and male (blue) patients without CLTI. Survival is given in years, reaching $98.6 \%$ at 1 year with no difference between the sexes. Panel B shows survival of female and male patients at the stage of CLTI being relevantly impaired (89\% at 1 year; p<0.001 compared to non-CLTI). After 4 years, survival rates were $92.5 \%$ in non-CLTI vs. $70.1 \%$ in CLTI ( $p<0.001)$. However, difference between the sexes did not reach statistical noticeability. (A) Non chronic limb threatening ischemia; (B) Chronic limb threatening ischemia.

discharge. After 12 months, OAC intake decreased in female and male patients ( $25 \%$ vs. $19 \%$ ). In contrast, $98 \%$ of patients received PAI at discharge, decreasing to $84 \%$ in female and $78 \%$ in male patients at 12 months followup visit. Moreover, male patients were more frequent on statin at index (66\% vs. $76 \%$ ) but statin usage improved at 6 months follow-up in both sexes reaching $87 \%$ of patients. It is noteworthy, that statin intake decreased again in female patients after 12 months to $79 \%$ adherence, but further improved in male patients (91\%). 
Factor

Age

Polyvascular disease*

CLTI**
Male

1.09

$(1.05 ; 1.13)$

1

$(0.54 ; 1.87)$

3.17

$(1.69 ; 5.96)$

4.73
$(1.03 ; 1.13)$

$(0.71 ; 4.08)$

$(1.33 ; 16.8)$

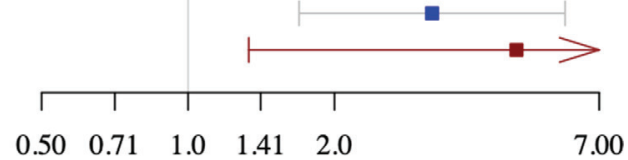

Figure 2. Adjusted long-term mortality of male and female LEAD patients. Cox regression analyzing 1 year mortality was performed in female (red) and male (blue) LEAD patients. The model was adjusted for the factors age, polyvascular disease, and CLTI and their impact on mortality is displayed as hazard ratio (HR) with 95\%- confidence intervals (CI). In both sexes, particularly CLTI was a noticeable predictor for mortality. In the combined model, HR for female sex was HR $0.755(0.437-1.303) p=0.312$ describing the impact of sex on long-term mortality. *Polyvascular disease - besides LEAD at least one further of the following: chronic coronary syndrome, cerebrovascular disease, visceral artery disease. ${ }^{* *} \mathrm{CLTI}$ - chronic limb threatening ischemia; LEAD Fontaine stage III and IV at index.

\section{Discussion}

We identified an uneven sex distribution in our university collective of LEAD patients receiving EVR. Whereas global epidemiological research suggests LEAD to be about equally prevalent in the male and female population [18], our university hospital setting displayed three-quarter of LEAD patients receiving EVR to be male. Even though German nationwide data support a preponderance of males among in-patient treated cases (66\% male LEAD cases in 2009; [19]), patients' sex is reported almost balanced among those who receive EVR on the large-scale (55\% male patients; [20]). A recent analysis on 41,873 LEAD patients found equal rates of overall revascularization procedures in both sexes and even a slight surplus of female patients regarding the endovascular approach [21]. Consistent with the greater picture drawn by claims data research $[19,20,21]$, female patients in our setting were comparably frequent at severe LEAD stages with critically reduced ABI values at index. Therefore, under the assumption that indication for EVR was placed irrespective of patients' sex, the under-representation of females cannot be plausibly explained by lower need for EVR. However, other university hospital settings likewise report on a high proportion of male patients receiving EVR that ranges between 61\% and $69 \%[22,23,24]$. In view of these data the question arises as to whether there may be fundamental barriers for female LEAD patients at the level of referral to gain access to high level medical expertise of university vascular centres. The access to guideline-recommended medicine has repeatedly been subject of discussion in the cardiovascular field $[25,26]$. In this context, female sex has previously been identified as a risk factor for underuse of guideline recommended medication in LEAD [27]. Correspondingly, statin intake was noticeable lower in female patients at admission and again at 12 months follow-up visits despite uniform recommendation at any stage of LEAD [28]. It can only be hypothesized why adherence to statins is reduced in the long term. Reasons may include conscious decision due to intolerance, refusal by the patient, or occurrence of potential contraindications, but also falling into oblivion in particular in patients not participating in subsequent ambulatory physician visits [29]. However, in contrast to current literature, we report equal medical supply with antithrombotic drugs (PAI and/or OAC) at index in $80 \%$ of LEAD patients and sustained high adherence up to 12 months.

In any case, with regard to the EVR, there were no relevant procedural differences detected between the two sexes. The usage of drug-based devices applied to almost every second patient (38\% DCB, $8 \%$ DES) reflecting the high technical availability of a university setting compared to the national average (4\% DCB, $1 \%$ DES; [20]). High primary technical success and sustained improvement of ABI values show EVR to be a good therapeutic option in either sex. Severe peri-procedural complications such as amputation $(3 \%)$, severe bleeding $(2 \%)$, or in-hospital mortality (1\%) are comparatively low in view of a cross-section of all health care suppliers (amputation 7\%, bleeding 10\%, death 2\%; [20]). Particularly severe adverse events (MCE; MALE) were observed at equal rates in female and male LEAD patients despite higher age in women. Observed survival of the entire cohort ranged around $95 \%$ at one year. As expected, survival is highly dependent on CLTI status with similar impact in male and female patients. However, even in the CLTI subset survival rates were exceedingly high with $89 \%$ at one-year and $70 \%$ at four-year FU in both sexes. This is in contrast to the nationwide supply situation where not only one-year survival rates are reported around 73\% in CLTI after EVR [30], but which also point at worse outcome in male patients reporting increased mortality (HR 1.155) and amputation risk (HR 1.284; [21]). Therefore, the current data of a specialized vascular centre may also indicate the quality of care that could be achieved under optimal conditions. Interestingly, the presence of polyvascular disease did not 
turn out as an independent risk factor of death neither in male nor in female patients.

\section{Limitations}

Our data depict a selection of LEAD patients that were assigned to our university vascular centre for EVR, and therefore results of the evaluation may underlie a selection bias. However, indication for EVR was examined and approved by specialized physicians utilizing standardized assessment of vascular status and patients' risk profiles. Therefore, relevant sex differences in the quality of consultation should be diminished as far as possible to the best of our knowledge. Second, assessment during follow-up is limited to those patients that participated in follow-up visits in our hospital. However, information on vital status is complete since this information was double-checked and updated in the form of comprehensive query by phone. Third, our conclusions are restricted to in-patient care, however patients' prognosis is relevantly depending on optimal ambulatory aftercare (e.g. regulation of pharmacotherapy, wound management). Last but not least, even though biological sex was object of our examination, aspects of gender such as socio-economic status, educational status, or living conditions may affect long-term results.

\section{Conclusion}

EVR can be performed with high technical success and low rate of severe complications both in male and female patients. In contrast to the national average, amputation rates and mortality at one year were equally reduced in either sex. However, in the specialized vascular centre, the preponderant part of patients receiving EVR is male, suggesting potential barriers for female LEAD patients to gain access to the medical supply of a university setting. Finally, analysis of pharmacotherapy indicates need for improvement involving tight integration of ambulatory aftercare. Further research is needed to shed light on medical sector overriding care in LEAD with focus on patients' sex.

\section{Electronic supplementary material}

The electronic supplementary material (ESM) is available with the online version of the article at https://doi.org/ 10.1024/0301-1526/a000978

ESM 1. Adverse events reported at 6 and 12 months FU visits (Table)

ESM 2. Distribution of ABI values of the treated side at index and 1 year after EVR for male and female patients (Figure)

ESM 3. Pharmacotherapy (Figure)

\section{References}

1. Criqui $\mathrm{MH}$, Aboyans V. Epidemiology of peripheral artery disease. Circ Res. 2015;116:1509-26.

2. Cacoub PP, Abola MTB, Baumgartner I, Bhatt DL, Creager MA, Liau CS, et al. Cardiovascular risk factor control and outcomes in peripheral artery disease patients in the Reduction of Atherothrombosis for Continued Health (REACH) Registry. Atherosclerosis. 2009;204:e86-92.

3. Wyss TR, Adam L, Haynes AG, Kucher N, Silbernagel G, Do DD, et al. Impact of cardiovascular risk factors on severity of peripheral artery disease. Atherosclerosis. 2015;242:97-101.

4. Song P, Rudan D, Zhu Y, Fowkes FJI, Rahimi K, Fowkes FGR, et al. Global, regional, and national prevalence and risk factors for peripheral artery disease in 2015: an updated systematic review and analysis. LancetGlobal Heal. 2019;7:e1020-30.

5. McDermott MM, Greenland P, Liu K, Criqui MH, Guralnik JM, Celic L, et al. Sex differences in peripheral arterial disease: leg symptoms and physical functioning. J Am Geriatr Soc. 2003;51:222.

6. Ramkumar N, Suckow BD, Brown JR, Sedrakyan A, Cronenwett JL, Goodney PP. Sex-based assessment of patient presentation, lesion characteristics, and treatment modalities in patients undergoing peripheral vascular intervention. Circ Interv. 2018;11:e005749.

7. Rieß HC, Debus ES, Heidemann F, Stoberock K, Grundmann RT, Behrendt CA. Gender differences in endovascular treatment of infrainguinal peripheral artery disease. VASA. 2017;46:296-303.

8. Roddy SP, Darling RC 3rd, Maharaj D, Chang BB, Paty PS, Kreienberg PB, et al. Gender-related differences in outcome: an analysis of 5880 infrainguinal arterial reconstructions. J Vasc Surg. 2003:37:399-402.

9. Schulte KL, Hardung D, Tiefenbacher C, Weiss T, Hoffmann U, Amendt $\mathrm{K}$, et al. Real-world outcomes of endovascular treatment in a non-selected population with peripheral artery disease - prospective study with 2-year follow-up. VASA. 2019;48:433-41.

10. Wawruch M, Wimmer G Jr, Murin J, Paduchova M, Tesar T, Hlinkova $L$, et al. Patient-associated characteristics influencing the risk for non-persistence with statins in older patients with peripheral arterial disease. Drugs Aging. 2019;36:863-73.

11. Ramkumar N, Suckow BD, Brown JR, Sedrakyan A, MacKenzie $\mathrm{T}$, Stone $\mathrm{DH}$, et al. Role of sex in determining treatment type for patients undergoing endovascular lower extremity revascularization. J Am Heart Assoc. 2019;8:e013088.

12. Hultgren R, Olofsson P, Wahlberg E. Sex-related differences in outcome after vascular interventions for lower limb ischemia. J Vasc Surg. 2002;35:510-6.

13. Eugster T, Gürke L, Obeid T, Stierli P. Infrainguinal arteria reconstruction: female gender as risk factor for outcome. Eur J Vasc Endovasc Surg. 2002;24:245-8.

14. Collaboration ABI, Fowkes FGR, Murray GD, Butcher I, Heald $\mathrm{CL}$, Lee RJ, et al. Ankle brachial index combined with Framingham Risk Score to predict cardiovascular events and mortality: a meta-analysis. JAMA. 2008;300:197-208.

15. Blonde L, Khunti K, Harris SB, Meizinger C, Skolnik NS. Interpretation and impact of real-world clinical data for the practicing clinician. Adv Ther. 2018;35:1763-74.

16. Reinecke H, Unrath M, Freisinger E, Bunzemeier H, Meyborg M, Lüders F, et al. Peripheral arterial disease and critical limb ischaemia: Still poor outcomes and lack of guideline adherence. Eur Heart J. 2015;36:932-8.

17. Aboyans V, Ricco JB, Bartelink MLEL, Björck M, Brodmann M, Cohnert T, et al. 2017 ESC Guidelines on the Diagnosis and Treatment of Peripheral Arterial Diseases, in collaboration with the European Society for Vascular Surgery (ESVS). Eur J Vasc Endovasc Surg. 2018;55:305-68.

18. Fowkes FG, Aboyans V, Fowkes FJ, McDermott MM, Sampson UK, Criqui MH. Peripheral artery disease: epidemiology and global perspectives. Nat Rev Cardiol. 2017;14:156-70. 
19. Malyar N, Fürstenberg T, Wellmann J, Meyborg M, Lüders F, Gebauer K, et al. Recent trends in morbidity and in-hospital outcomes of in-patients with peripheral arterial disease: a nationalwide population-based analysis. European Heart Journal. 2013;24:2706-14.

20. Freisinger E, Koeppe J, Gerss J, Goerlich D, Malyar NM, Marschall $U$, et al. Mortality after use of paclitaxel-based devices in peripheral arteries: a real-world safety analysis. Eur Heart J. 2020;41:3732-9.

21. Freisinger E, Malyar NM, Reinecke H, Unrath M. Low rate of revascularization procedures and poor prognosis particularly in male patients with peripheral artery disease - A propensity score matched analysis. Int J Cardiol. 2018;255:188-94.

22. Brodmann M, Moscovic M, Wang JCC, Nano G, Dahm J, Zeller T, et al. Real-world experience with a paclitaxel-coated balloon in critical limb ischemia: 24-month subgroup outcomes of BIOLUX P-III. JACC Cardiovasc Interv. 2020;13:2289-99.

23. Krankenberg $H$, Tübler $T$, Sixt $S$, Fischer M, Schmiedel R, Schulte KL, et al. German multicenter real-world registry of stenting for superficial femoral artery disease: clinical results and predictive factors for revascularization. J Endovasc Ther. 2014:21:463-71.

24. Schulte KL, Hardung D, Tiefenbacher C, Weiss T, Hoffmann U, Amendt $\mathrm{K}$, et al. Real-world outcomes of endovascular treatment in a non-selected population with peripheral artery disease - prospective study with 2-year follow-up. VASA. 2019;48:433-41.

25. Reinecke $H$, Unrath M, Freisinger E, Bunzemeier H, Meyborg M, Lüders F, et al. Peripheral arterial disease and critical limb ischaemia: still poor outcomes and lack of guideline adherence. European Heart Journal. 2015;36:932-8.

26. Vemulapalli S, Greiner MA, Jones WS, Patel MR, Hernandez AF, Curtis LH. Peripheral arterial testing before lower extremity amputation among Medicare beneficiaries, 2000 to 2010. Circ Cardiovasc Qual Outcomes. 2014;7:142-50.

27. Makowski L, May D, Hörter S, Ranft J, Malyar N, Gebauer K, et al. Sex related differences in patients with peripheral artery disease - German Health Claims Data. Clin Res Cardiol. 2019;108(Suppl 1). Available from https://dgk.org/kongress_ programme/jt2019/aV218.html

28. Deutsche Gesellschaft für Angiologie - Gesellschaft für Gefässmedizin, Arbeitsgemeinschaft der Wissenschaftlichen
Medizinischen Fachgesellschaften (AWMF). Deutsche Gesellschaft für Angiologie - Gesellschaft für Gefässmedizin, Arbeitsgemeinschaft der Wissenschaftlichen Medizinischen Fachgesellschaften (AWMF). S3-Leitlinie zur Diagnostik, Therapie und Nachsorge der peripheren arteriellen Verschlusskrankheit. AWMF-Register Nr. 065/003 Entwicklungsstufe 3. 2015. Verfügbar unter https://www.awmf.org/uploads/tx_szleitlinien/ 065-003L_S3_PAVK_periphere_arterielle_Verschlusskrankheit_ 2020-05.pdf

29. Bansilal S, Castellano JM, Garrido E, Wei HG, Freeman A, Spettell $C$, et al. Assessing the impact of medication adherence on long-term cardiovascular outcomes. J Am Coll Cardiol. 2016;68:789-801.

30. Stella J, Engelbertz C, Gebauer K, Hassu J, Meyborg M, Freisinger $E$, et al. Outcome of patients with chronic limbthreatening ischemia with and without revascularization. VASA. 2020;49:121-7.

\section{History}

Submitted: 14.06.2021

Accepted after revision: 27.10.2021

Published online: 29.11.2021

\section{Conflict of interest}

The authors declare that there are no conflicts of interest.

\section{Authorship}

We declare that all authors participated in the research and preparation of the manuscript.

\section{Correspondence address}

Eva Freisinger, MD

Division of Vascular Medicine

Department of Cardiovascular Medicine

University Hospital Muenster

Albert Schweitzer Campus A1

48149 Muenster

Germany

eva.freisinger@ukmuenster.de 